\title{
Meshless Numerical Solution of Boundary Integral Equations based on Non Uniform Rational Basis-Splines
}

\author{
Vincenzo Marchese and Umberto Iemma \\ Department of Engineering, Roma Tre University, via Vito Volterra, 62, Roma, Italy
}

\begin{abstract}
(Received 15 August 2013; accepted 11 December 2014
The paper deals with the use of Non Uniform Rational Basis-Splines (NURBS) for the global representation of domain geometry and unknown functions aimed at the numerical solution of Boundary Integral Equations (BIE). The use of a global NURBS function basis yields a meshless method which does not need the partition of the boundary into elements. The level of the accuracy in the representation of dependent and independent variables can be changed in each simulation, according to the problem requirements, thanks to the recursive definition of NURBS. The solving system of equations is assembled by means of the collocation of the integral equation onto the Greville abscissae in the NURBS parametric space. The unknowns are the locations of the control points in the vector space the unknown function belongs to. Preliminary numerical results have been obtained in potential aerodynamics and acoustic scattering. The numerical solution reveals a remarkable level of accuracy in all the test cases analyzed with a convergence rate always higher than the order of the NURBS adopted.
\end{abstract}

\section{INTRODUCTION}

In many fields of application, the Boundary Integral Equations (BIE) approach is a well established technique to address the solution of Boundary Value Problems (BVP). Using the BIE, it is possible to represent the unknown function at any location in the domain as a function of its Cauchy data set. This approach is more recently considered as standard in incompressible and compressible potential aerodynamics, structural elasticity, heat conduction, electromagnetism, acoustics, and aeroacoustics. The numerical solution typically relies on the Boundary Element Method (BEM), in all its variants and declinations. In classic BEM the boundary of the domain is partitioned into finite elements, where the dependent and independent variables are approximated using suitable local basis functions. The greatest advantage of the numerical methods based on BIE resides in the reduction of the computational burden required for the numerical solution, due to the reduced dimensionality of the problem. Although this approach is usually convenient with respect to the so-called field methods, such as Finite Volumes Method (FV), Finite Difference Method (FDM), or Finite Element Method (FEM), in some specific application the number of boundary elements required to capture a specific feature of the phenomenon may become extremely high. This is the case, for example, for acoustic propagation and scattering problems, where the wave length of the perturbation at high frequencies can be orders of magnitude smaller than the characteristic length of the domain of interest, thus requiring a huge amount of boundary panels to correctly reproduce the scattering and interference effects. One of the possible approaches to mitigate this difficulty is improving the accuracy of the local representation of the variables using higher-order functions. This allows for the reduction of the number of elements needed to achieve the desired level of accuracy. In this respect, the literature available is very extensive, and an in-depth review is beyond the scope of the paper. Among others, it is worth mentioning the use of third order polynomials based on Overhauser ${ }^{1,2}$ or Hermite ${ }^{3,4}$ elements, recently coupled with Coons patches. ${ }^{5}$ The typical limitation of all the approaches based on the local representation of the variables is the restriction of the resulting numerical formulation to a single order of accuracy, fixed by the order of the polynomial shape functions used. The possibility to overcome this limitation has already been investigated within the context of the finite element method $^{6}$ using an approach based on generalized Hermite polynomials. However, a similar attempt for the solution of BIE is still missing. This goal has been the driving motivation in the development of the method presented here. Indeed, using a global representation of the variables based on NURBS, the order of the basis functions can be improved when needed by the specific application in discussion. This is a consequence of the iterative definition of the NURBS, which makes possible the increase of the NURBS degree simply by changing an input parameter. The use of the NURBS for the representation of curves and surfaces is a common technique in the CAD community for the modeling of complex geometries with strict requirements of smoothness and continuity between patches. ${ }^{7,8}$ Their use in the numerical solution of BIE is not new, although it is relatively recent. NURBS have been used to develop boundary element solutions of integral equations in elastostatics, ${ }^{9}$ in radiation and diffraction problems, ${ }^{10}$ and in potential aerodynamics. ${ }^{11}$ As already mentioned, the peculiarity of the present method is in the use of the NURBS for the development of a global isogeometric approach aimed at the meshless numerical solution of the BVP. The control points used for the representation of the dependent variables are obtained through the $h$-refinement of the optimal NURBS 